\title{
The Contact of Graphene with Ni(111) Surface: Description by Modern Dispersive Forces Approaches
}

\author{
Helena Muñoz-Galán, ${ }^{1}$ Francesc Viñes, ${ }^{1,}{ }^{*}$ Julian Gebhardt, ${ }^{2}$ Andreas Görling, ${ }^{2}$ and Francesc Illas ${ }^{1}$ \\ ${ }^{I}$ Departament de Química Física \& Institut de Química Teòrica i Computacional (IQTCUB), Universitat de \\ Barcelona, c/Martí i Franquès 1, 08028 Barcelona, Spain.
}

${ }^{2}$ Lehrstuhl für Theoretische Chemie, Universität Erlangen-Nürnberg, Egerlandstr. 3, 91058 Erlangen, Germany.

"Corresponding author: francesc.vines@ub.edu

\begin{abstract}
Here we present a Density Functional Theory (DFT) study on the suitability of modern corrections for the inclusion of dispersion related terms $(D F T-D)$ in treating the interaction of graphene and metal surfaces, exemplified by the graphene/Ni(111) system. The Perdew-Burke-Ernzerhof $(P B E)$ exchange-correlation functional is used as basis, on top of which we tested the family of Grimme corrections (D2 and D3, including Becke-Jonson damping and Andersson approach) as well as different flavors of the approach by Tkatchenko and Scheffler (TS). Two experimentally observed chemisorbed states, top-fcc and bridge-top conformations, were examined, as well as one physisorbed situation, the hcp-fcc state. Geometric, energetic, and electronic properties were compared to sets of experimental data for our model system of graphene/Ni(111), but also for available data of bulk Ni, graphite, and free-standing graphene. Results show that two of the most recent approximations, the fully ab initio TS-MBD, and the semi-empirical Grimme D3 correction are best suited to describe graphene $\leftrightarrow$ metal contacts, yet, comparing to earlier studies, the Rev-vdWDF2 functional is also a good option, whereas optB86-vdW and optB88b-vdW functionals are fairly close to experimental values to be harmless used. The present results highlight how different approaches for the approximate treatment of dispersive forces yield different results, and so finetuning and testing of the envisioned approach for every specific system is advisable. The present survey clears the path for future accurate and affordable theoretical studies of nanotechnologic devices based on graphene-metal contacts.
\end{abstract}

Keywords: Graphene $\cdot$ Dispersive Forces $\cdot$ Ni(111) $\cdot$ Density Functional Theory 


\section{Introduction}

Since graphene was isolated in 2004 by Geim and coworkers [1], it has been rapidly gaining importance to become a hub of nanotechnological electronics research. Electronic and technological industries have focused on graphene as its mechanical and electric properties, combined with an, in theory, low fabrication cost, make it a promising substitute for conventional materials. For the synthesis of high-quality and large-size patches of graphene for transparent electronics and high frequency devices industry a variety of methods evolved, including exfoliation [1], epitaxial growth [2-4], and chemical vapor deposition [5-7].

$\mathrm{Ni}(111)$ quickly gained momentum against other metals in graphene synthesis since the lattice mismatch of graphene with respect to $\mathrm{Ni}(111)$ is propitiously small allowing the formation of well-ordered, rather large, patches [8]. This makes Ni(111) suitable for systematic studies of graphene adsorption as well. In addition, conduction measurements involving graphene need metal electrodes to be carried out, so a full understanding of the physics of graphene with metal surfaces is essential. Fortunately, the binding mechanism has been addressed by a variety of theoretical and experimental studies and is now well understood. Previous studies based on X-Ray Photoemission Spectroscopies (XPS) detected two energetically degenerate attachment conformations of graphene on $\mathrm{Ni}(111)$ [9], the so-called bridge-top and top-fcc chemisorbed conformations. The possible identification of these arrangements by Scanning Tunneling Microscopy (STM) was suggested from theoretical predictions in that study and confirmed in a subsequent study by high-resolution STM [10]. Other conformations, such as hcp-fcc, imply that graphene is detached (physisorbed) over the Ni(111) surface [9]. The top-hcp conformation, despite being experimentally considered as a possibility similar to top-fcc [10], has been characterized as a transition state in between conformations [9].

Due to its particular electronic structure, graphene is a zero bandgap semimetal and exhibits the famous linear band dispersion in the vicinity of each k-point within the Brillouin zone.[11,12] However, these Dirac points may be strongly modified when graphene adsorbs on metal surfaces, as shown in previous studies [2,5,13]. Indeed, thorough theoretical calculations show strong hybridization between metal bands and the graphene $\pi$-band for bridge-top and top-fcc modes $[14,15]$, breaking the linear dispersion at the Dirac point. In contrast, in the weakly physisorbed hcpfcc graphene conformation, the $\pi$-band does not strongly interact with the Ni(111) bands due to the larger adsorption distance, and, consequently, the linear dispersion prevails. In that case, the 
influence of the substrate results only in a small shift of the Dirac point with respect to the Fermi level, i.e., a small doping effect [16].

Due to the variety of different bonding motives including chemisorption and physisorption, as well as the importance of the graphene/Ni(111) system, it constitutes a textbook example of metal $\leftrightarrow$ graphene interactions, results of which can be extended to other transition metals. Previous theoretical and experimental studies showed that dispersive forces play a crucial role in this system, just as in the adsorption of aromatic molecules on metal surfaces $[15,17,18]$.

Initially ab initio Density Functional Theory $(D F T)$ studies relied on the Local Density Approximation $(L D A)$, which could apparently correctly discern between physisorbed and chemisorbed states [16]. Nowadays this is known to happen because of the LDA exchangecorrelation functionals tend to overbind [19]. The upgrade to Generalized Gradient Approximation (GGA), despite getting rid of part of the overestimation, does not help in here due to the missing description of long range dispersive interactions. Indeed GGA functionals yield adverse adsorption energies of graphene [20], in the sense that a system where graphene is fully separated to the metal surface is energetically preferred. This is also the case when using meta-GGA functionals, yet to a lesser extent [21]. Hybrid functionals, providing excellent description of the thermochemistry of main group molecules, are unadvised, because of their failure in treating largely delocalized systems, such as transition metals and graphene [22]. Calculations within the Random Phase Approximation $(R P A)$ were suggested as a good choice [23] to model graphene-metal systems. However, on the one hand, these are computationally too expensive for many practical systems, and, on the other hand, RPA yields physisorbed and chemisorbed situations with similar adsorption energy, for which there is no experimental evidence, i.e. it appears that RPA overestimates the binding strength for physisorbed situations.

Thus, the best approach to describe graphene/ $\mathrm{Ni}(111)$ and similar systems is to employ GGA DFT including dispersion terms through one of the currently available methods to cure the lack of long range dispersive interactions. Many functionals and corrections evolved over the last decade, since more sophisticated approaches are still computationally limited for such a system and ad hoc solutions like single-shot, i.e. non self-consistent RPA seem not to be successful for this challenging example.

Most of (semi-empirical) van der Waals corrections $(v d W)$ can be categorized into two different approaches, namely the $i$ ) (non-local) van der Waals functionals (vdW-DFT) and ii) energy corrections, aimed at accounting for dispersion terms, directly added to the ground state energy 
determined by standard GGA functionals (DFT-D methods). The validation of such newly developed vdW-DFT functionals or DFT-D corrections has been matter of recent investigations [8,24]. However, a systematic investigation, with a fair comparison of methodologies, is still lacking in this evolving field. We herein provide a systematic assessment of the recent flavors of the most successfully applied DFT-D methods of Grimme [25] and Tkatchenko [26]. To this end, we chose the graphene/Ni(111) system already described, comparing structure, energy, and bandstructure of the physisorbed and chemisorbed conformations - bridge-top and top-fcc- obtained by these methods, relating these results to earlier works, spanning a wide range of vdW-DFT functionals and DFT-D corrections.

\section{Computational details}

Three adsorption conformations for graphene adsorbed on the Ni(111) surface have been studied; namely the so-called top-fcc and bridge-top chemisorbed situations, and the hcp-fcc physisorbed situation, see Fig. 1. A representative GGA functional, the Perdew-Burke-Erzenhof $(P B E)$ [27], has been used as the basis for the studied DFT-D corrections, given its appropriate description of transition metals [19] as well as of graphene [8] and, in particular, matching the interatomic experimental distance of bulk Ni. Spin-polarized DFT calculations were performed with the VASP 5.3.5 calculation package [28], using the Projector Augmented Wave $(P A W)$ method to treat core electrons and their interaction with valence electrons [29]. A plane-wave basis set has been used with kinetic energy cut-off of $415 \mathrm{eV}$, which has been found to yield optimized results in previous works $[8,9,19]$. The reciprocal space has been sampled with a $7 \times 7 \times 1$ Monkhorst-Pack [30] k-point grid, bandstructures have been obtained using a $9 \times 9 \times 1$ k-points grid. Geometry optimizations have been considered converged once forces acting on relaxed atoms have become smaller than $0.03 \mathrm{eV} \AA^{-1}$. The tetrahedron smearing by Blöchl was used for the electronic convergence [31].

All tested vdW corrections are based on pair-wise interactions dependent on $\mathrm{C}^{6}$ coefficients and atomic radii $\mathrm{R}_{0}$ for each species, which is damped by some function for small interatomic distances, see Ref. [25] for details. The Grimme D2 correction was later re-parameterized, with $\mathrm{C}_{6}$ coefficients becoming geometry-dependent, at the toll of adding new adjustment parameters $\mathrm{S}_{6}, \mathrm{~S}_{8}$, and $S_{R}$ [32]. In addition, damping the vdW contribution not to zero but a finite small value, as proposed by Becke-Jonson (BJ), was introduced [33]. Finally, another modification was proposed later by Andersson (A), claiming to replace the $\mathrm{C}_{6}$ parameters of transition metals by that of the noble gas of the upper row in the periodic table [34], in addition to tightening the valence electron screening. 
An alternative approach was presented by Tkatchenko and Scheffler (TS). Formally TS is based on $\mathrm{D} 2$, seeking, however, to evaluate $\mathrm{C}_{6}$ and $\mathrm{R}_{0}$ for arbitrary systems from the respective coefficients of free atoms via the evaluation of the electron density within the studied system [26]. Afterwards, the TS correction evolved to include the polarizability variation due to the electric field change of surrounding polarizable atoms in the Self Consistent Screening (SCS) TS-SCS method [35]. Lately, the TS Many Body Dispersion (MBD) TS-MBD has been proposed to improve the description of the long-range many body nature of correlation and dispersion interactions [36]. Note however that for TS methods, surface adapted $\mathrm{C}^{6}, \alpha$ atomic polarizability, and $\mathrm{R}_{0}$ parameters [37] are necessary for the description of transition metal substrates. This is, the reference state in TS surf is changed from free-atoms to an atoms-in-bulk situation [38]. Note in passing that the alternative, of including vdW effects by one-electron potentials is here not considered [39].

A six layer $\mathrm{p}(1 \times 1) \mathrm{Ni}(111)$ slab unit cell was used in the calculations, where three bottom layers were fixed to PBE bulk-optimized positions - targeting the experimental Ni positionswhereas the three top layers were relaxed - the so-called $3+3$ approximation. Note that previous calculations showed very little variations on graphene adsorption energetics and structure by using Ni bulk positions as optimized by the method under scrutiny instead of those obtained by PBE, as working vdW schemes should yield similar values [8]. The adjacent slabs in the direction perpendicular to the surface were separated by a vacuum width of $1 \mathrm{~nm}$. The adsorption energy of graphene on $\mathrm{Ni}(111), E_{a d s}$, given per $\mathrm{C}$ atom, was calculated as:

$$
E_{a d s}=\left[-E_{G r / N i}+\left(E_{N i}+E_{G r}\right)\right] / 2
$$

where 2 is the number of carbon atoms in the used unit cell, $E_{G r / N i}$ is the total energy of graphene attached to the $\mathrm{Ni}(111)$ slab, $E_{N i}$ that of the pristine $\mathrm{Ni}(111)$ slab, and $E_{G r}$ that of free-standing graphene. Within this definition, the more positive the adsorption energy is, the more exothermic the adsorption. The graphene distance with respect to the $\mathrm{Ni}(111)$ surface, $d(\mathrm{Gr}-\mathrm{Ni})$, has been calculated from the mean plane of the graphene sheet and the Ni(111) surface plane, respectively. The isolated graphene reference has been calculated using the same unit cell by just removing the Ni layers while allowing graphene contraction/expansion, yet maintaining a minimum vacuum width of $1 \mathrm{~nm}$.

\section{Results and discussion}

At first, the mismatch between a free-standing graphene sheet and the Ni(111) surface was briefly evaluated. The lattice parameter $(a)$ of graphene and interatomic distance $(a)$ of $\mathrm{Ni}(111)$ are listed in Table S1 in the Supplementary Material. The results therein show that all studied methods are well suited to describe the graphene lattice, with errors of, at most, $0.4 \mathrm{pm}$. In the case of the Ni 
bulk, the interatomic distance is almost in perfect agreement with experiment for PBE, D2, and D3. The D3-BJ or A modification yield a small contraction by up to $2.5 \mathrm{pm}$. The TS based methods results in slightly more acute underestimations of at most $5.8 \mathrm{pm}$. In summary, the lattice mismatch of $\sim 1 \%$ is correctly described applying D2 or D3, with the exception of D3-BJ and A modifications, which yield no mismatch. The TS based method performs reasonably well but with an opposite mismatch in between -0.3 and $-1.3 \%$.

Having addressed the graphene and $\mathrm{Ni}(111)$ systems separately, the graphene layer has been optimized on top of the $\mathrm{Ni}(111)$ slab in bridge-top, top-fcc, and hcp-fcc positions. The adsorption energies $E_{a d s}$ and the graphene $\leftrightarrow \mathrm{Ni}$ distances, $d(\mathrm{Gr}-\mathrm{Ni})$, are listed in Table S2 of the Supplementary Material. The obtained data are compared to accurate experimental values yielding a graphene $\leftrightarrow \mathrm{Ni}(111)$ distance of $211 \pm 7 \mathrm{pm}$ [40] and precise adsorption energy values derived from graphite attachment to $\mathrm{Ni}(111)$ [41] and the graphite exfoliation energy [42], which yield an experimental value of $9.2 \pm 2.0 \mathrm{~kJ} \mathrm{~mol}^{-1}$ per $\mathrm{C}$ atom [8], yet some margin of error is attributable to this graphene attachment energy, and so, comparison should be made with a broad perspective, lacking experimental data with improved accuracy.

Fig. 2 graphically shows the accuracy of the tested methods computing the Mean Error $(M E)$ with respect to the mean experimental adsorption energy, including the limits of experimental uncertainty. Note that experimentally bridge-top and top-fcc are detected by XPS [9], or observed by STM [10], although a particular preference of one against the other is not clear, so they should be considered as essentially isoenergetic. Nevertheless, a small preference of bridge-top was suggested by XPS data, although this claim must be kept with great caution. This is well observed in Fig. 2; the adsorption energy difference between bridge-top and top-fcc is small for all methods, varying from $0.1 \mathrm{~kJ} \mathrm{~mol}^{-1}$ per $\mathrm{C}$ atom (PBE) to $1 \mathrm{~kJ} \mathrm{~mol}^{-1}$ per $\mathrm{C}$ atom (A).

Concerning the accuracy of the tested DFT-D methods, PBE clearly underestimates the strength of the graphene/Ni(111) interaction, in line with previous results [8] and inherent to PBE due to the neglect of dispersion terms. As far as $\mathrm{vdW}$ corrections on PBE are concerned, it is to highlight the excellent performance of TS-MBD, well within the experimental $E_{a d s}$ values, closely followed by D3 correction. Former approximations, i.e. D2 and TS yield adsorption energies close to the experimental thresholds, but faintly overbinding, up to 1.8 and $1.2 \mathrm{~kJ} \mathrm{~mol}^{-1}$ per $\mathrm{C}$ atom, respectively. The $\mathrm{BJ}$ and $\mathrm{A}$ corrections yield a more acute over- and underestimation of the interaction, by up to 4.1 and $-2.7 \mathrm{~kJ} \mathrm{~mol}^{-1}$. The most striking, however, is the overestimation of TSSCS by almost $40 \mathrm{~kJ} \mathrm{~mol}^{-1}$. Note however that such an overestimation can be rationalized in the sense that long-range screening in TS-SCS leads to an anisotropic polarization of the electron density 
[35], which is counteracted in three-dimensional isotropic bulk systems, but does not in anisotropic systems such as surfaces, leading to this large overestimation.

Because of this, TS-SCS is suited in describing bulk graphite, as seen in Table S3 of Supplementary Material [43], with exfoliation energies within the experimental range, only $0.3 \mathrm{~kJ}$ $\mathrm{mol}^{-1}$ far from previous TS-SCS calculations [35], and small variations of $0.4 \mathrm{pm}$ for $a$ cell parameter and $6 \mathrm{pm}$ for interlayer distance with respect the experimental structure. Current calculations at TS and TS-MBD yield exfoliation energies slightly above the experimental range by 3.5 and $1.3 \mathrm{~kJ} \mathrm{~mol}^{-}$ ${ }^{1}$, although previous calculations with finer k-point mesh and larger plane-wave basis set show that this discrepancy is reduced by $\sim 2 \mathrm{~kJ} \mathrm{~mol}^{-1}[44,45]$ and variation with respect graphite interlayer distance of $\sim 5 \mathrm{pm}$. Overall, any of the here tested methods is well suited in describing bulk graphite, with the caveat of $\mathrm{PBE}$, which yields negative exfoliation energies and interlayer distances overestimated by more than $20 \mathrm{pm}$, due to the lack of dispersive forces description, and BJ damping, which yields exfoliation energies more than $14 \mathrm{~kJ} \mathrm{~mol}^{-1}$ larger than the experimental values, plus interlayer distances overestimated by $\sim 8 \mathrm{pm}$.

Back to graphene on $\mathrm{Ni}(111)$, note that, as observed in Table S2, the physisorbed hcp-fcc conformation is correctly described by any of the DFT-D corrections as a weaker attached situation, typically about $\sim 4-10 \mathrm{~kJ} \mathrm{~mol}^{-1}$ per $\mathrm{C}$ atom weaker. No physisorbed state has been experimentally observed for graphene on $\mathrm{Ni}(111)$, and has only been foreseen from the theoretical point of view, i.e., this situation must be energetically less favorable than any of the bridge-top or top-fcc chemisorbed situations. This is an indication for the failure of the non self-consistent treatment of graphene/Ni(111) within the RPA, yielding almost isoenergetic physisorbed and chemisorbed states $[23,46]$.

Next, we discuss the ME values of the graphene/Ni(111) attachment distance, shown in Fig. 3. It is known that DFT methods usually provide excellent structural data, providing interatomic distances within good accuracy, whereas energetics is more difficult to describe [19,22]. The DFT-D methods tested here are no exception and all methods can be considered within experimental accuracy — note that top-fcc case in D3-BJ targets the mean experimental value, and bridge-top case of TS-SCS and TS-MBD gives a distance just 2 and 1 pm below the experimental range- . Bridgetop conformation distances are usually lower than the average experimental value or computed distances for the top-fcc arrangement. Considering the physisorbed situation hcp-fcc, all methods yield a $d(\mathrm{Gr}-\mathrm{Ni})$ distance above $300 \mathrm{pm}$, as typically observed for the physisorbed states of graphene on noble metals, such as $\mathrm{Au}[5]$. 
We now focus on the band structure of graphene/ $\mathrm{Ni}(111)$ comparing the energy levels $\varepsilon$ of the graphene $\sigma$ and $\pi$ bands at $\boldsymbol{\Gamma}$ and $\mathbf{K}$ points of reciprocal space to experimental Angle-Resolved Photoemission Electron Spectroscopy $(A R P E S)$ data [9,47] (see Table S4 of the Supplementary Material). More precisely, we compare $\sigma$ and $\pi$ bands at $\Gamma$, and only the $\sigma$ band at $\mathbf{K}$, since the $\pi$ band around $\mathbf{K}$, i.e., the Dirac point, is disturbed as above mentioned. An exemplary bandstructure is shown in Fig. 4. Despite the sensitivity of $E_{a d s}$ with respect to the applied vdW correction, the bandstructure is well reproduced by any of the tested methods. This is in line with earlier reports demonstrating that the relative level of graphene to substrate bands is determined by the adsorption distance, which only showed small deviations throughout the variety of tested methods [8]. It is worth noting that $\varepsilon_{\sigma}^{\Gamma}$ usually is in very good agreement with experiment, with overall deviations of at most $0.1 \mathrm{eV}$. This deviation is increased when comparing eigenstates lower in energy, with deviations of up to 0.5 and $0.8 \mathrm{eV}$ for $\varepsilon_{\pi}^{\Gamma}$ and $\varepsilon_{\sigma}^{\mathrm{K}}$, respectively. Fig. 4 also shows the well-known opening of the Dirac points caused by the graphene $\leftrightarrow \mathrm{Ni}(111)$ interactions. Last but not least, the physisorbed state for graphene on $\mathrm{Ni}(111)$, would it exist, would feature $\varepsilon_{\sigma}^{\Gamma}, \varepsilon_{\pi}^{\Gamma}$, and $\varepsilon_{\sigma}^{\mathrm{K}}$ eigenvalues at $\sim 3,9$, and $10 \mathrm{eV}$ respectively, as similarly found for graphene on intercalated $\mathrm{Au}$ monolayer on $\mathrm{Ni}(111)[5]$.

At this point one could try to assess the suitability of DFT, DFT-D, vdW-DFT, and RPA methods in describing the interaction of graphene with $\mathrm{Ni}(111)$ surface in particular, and on transition metals in general. To discern over the archipelago of data found in the literature, the topfcc conformation has been chosen, as it happens to be a conformation in common for the full set of literature, see Table S2 in Supplementary Material. A comparison for $E_{a d s}$ is given in Fig. 5, whereas structural data analyzed in terms of $d(\mathrm{Gr}-\mathrm{Ni})$ is provided in Fig. 6. Note beforehand that one must be indulgent in such a comparison since different computational setups were used for the calculations, with different k-point grid density, plane wave kinetic energy cutoff, or different types of basis sets and pseudopotentials, to name a few, and present tests reveal that these factor may vary $E_{a d s}$ by 1-2 $\mathrm{kJ} \mathrm{mol}^{-1}$ and $d(\mathrm{Gr}-\mathrm{Ni})$ by 2-3 pm. However, general trends can be captured and discussed; see for instance the PBE results, where present and past results [8,48] essentially coincide; this is also the case for D2 and D3 $E_{a d s}$ values. A caveat is necessary for the D3 results obtained by Li and coworkers [49], reporting a slightly weaker adsorption, and larger $d(\mathrm{Gr}-\mathrm{Ni})$ distances, as shown in Figs. 5 and 6. The authors apparently found the physisorbed state of graphene on top-fcc conformation by D3, whereas PBE and other vdW-DFT found the proper chemisorbed situation. Note that a physisorbed state exists above the chemisorbed ones $[9,14,15]$, and this is exactly the case found for other vdW-DFT methods, such as the revPBE-vdW, rVV10, vdW-DF, vdW-DF2, and 
vdW-WFs1-shift — we address the reader to the original papers for further details-. All that said, we may now determine which methods provide results within the experimental accuracy for both $E_{a d s}$ and $d(\mathrm{Gr}-\mathrm{Ni})$. Neglecting the physisorbed situation of Li and coworkers [49], there are only three methods fulfilling these criteria: The TS-MBD and Grimme D3 methods, and the Rev-vdWDF2 [50], which relies on using the Becke-86 GGA functional [51] for the vdW-DF2 functional [52]. Note aside that, as previously stated [8], the optB86b-vdW [53] and optB88b-vdW [54] functionals

yield energetic data very close to the experimental reported ones, only a few tenths of $\mathrm{kJ} \mathrm{mol}^{-1}$ away from experimental value limits, and so could be fairly used to investigate this type of systems.

\section{Conclusions}

To sum up, the performance of modern DFT-D corrections - Grimme D2, D3, BJ damping, and A corrections, as well as TS, TS-SCS, and TS-MBD - applied in conjunction with PBE has been assessed relying on their description of the adsorption of graphene on $\mathrm{Ni}(111)$ featuring chemisorbed and physisorbed states, as a paradigm for adsorption on late transition metals. Two experimentally observed chemisorbed states, namely top-fcc and bridge-top, were examined, as well as a hypothetic physisorbed situation (hcp-fcc). Geometric, energetic, and electronic properties of graphene adsorbed on $\mathrm{Ni}(111)$, bulk $\mathrm{Ni}$, graphene, and graphite were compared to sets of experimental data. From the results it is clear that all methods are suited to describe graphene $\leftrightarrow$ metal contacts, with the exception of TS-SCS, which yields a sensible binding strength overestimation due to a long-range screening anisotropic polarization.

A survey of present tested methods compared to previous DFT-D corrections, vdW-DFT functionals, as well as RPA shows that fully ab initio TS-MBD and semi-empirical Grimme D3 corrections, as well as the Rev-vdW-DF2 functional, are best suited to describe graphene/Ni(111) system providing chemical accuracy, although optB86b-vdW and optB88b-vdW vdW-DFT functionals are fairly close. Note that these results are likely to hold for the adsorption of graphene on other transition metals. However, the suitability of each method should be further validated on other metals with detailed experimental data and a restricted testing of the here presented methods. Present results highlight how different approaches to introduce dispersion in DFT based methods may yield discrepant results, mostly due to the subtle treatment of such a weak interaction. In any case, one must take dispersion related interactions with great caution when studying systems of technologic interest. Along this line, the present survey clears the path for future accurate and affordable theoretical studies of nanotechnologic devices based on graphene-metal contacts. 
Acknowledgments This work has been supported by Spanish MINECO CTQ2012-30751 and CTQ2015-64618-R grants, in part by Generalitat de Catalunya grants 2014SGR97 and XRQTC, and by the Deutsche Forschungsgemeinschaft through SFB 953. This project has received funding from the EU Horizon 2020 research under the NOMAD Excellence Center (grant Number 676580). Authors are greatly indebted to Alexander Tkatchenko and Thomas Bučko for fruitful discussion. F.V. thanks MINECO for a postdoctoral Ramón y Cajal (RyC) research contract (RYC-2012-10129). F.I. acknowledges additional support from the 2015 ICREA Academia Award for Excellence in University Research. 
Fig. 1 Side and top views on graphene adsorbed on Ni(111) in bridge-top (left), hcp-fcc (middle), and top-fcc (right) conformations. Violet spheres represent carbon atoms, whereas nickel atoms are colored with diverse tones to differentiate Ni layers.

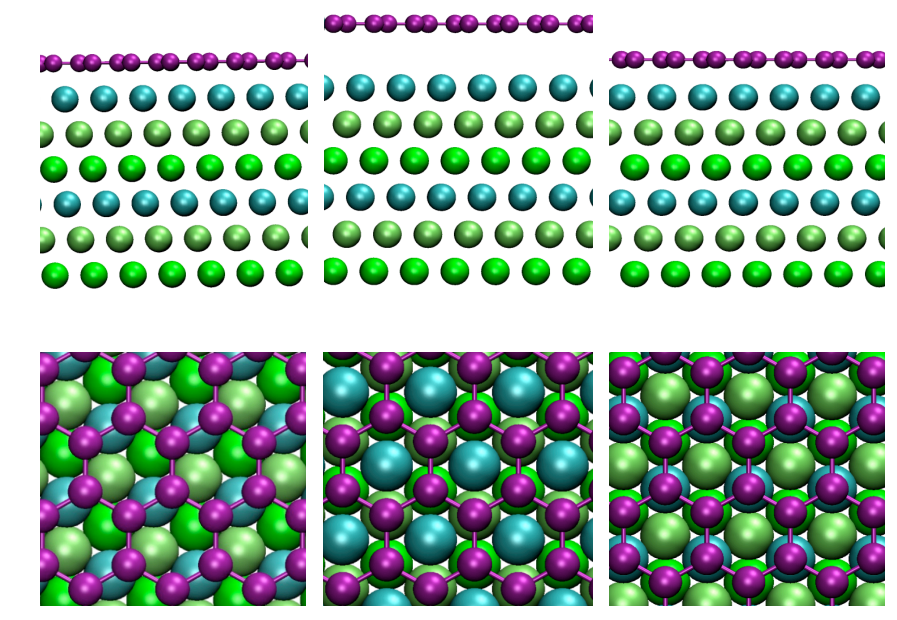


Fig. 2 Mean Error $(M E)$ with respect to averaged experimental results, in $\mathrm{kJ} \mathrm{mol}^{-1}$, of the adsorption energy $\left(E_{a d s}\right)$ of graphene on $\mathrm{Ni}(111)$ at bridge-top (blue bars) and top-fcc (green bars) for PBE and various DFT-D schemes. Dashed horizontal lines indicate the experimental deviation.

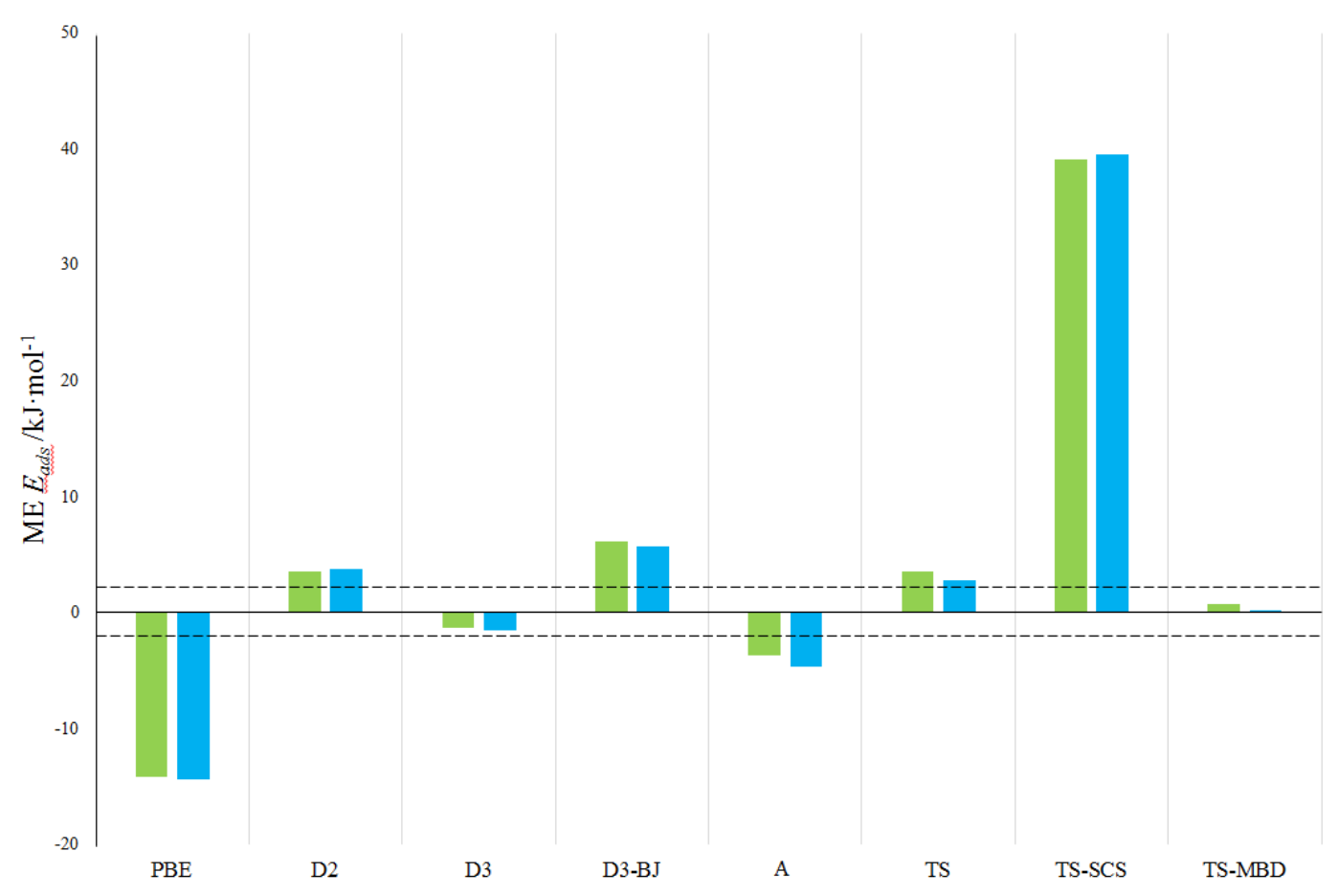


Fig. 3 Mean Error $(M E)$, in pm, for $d(\mathrm{Gr}-\mathrm{Ni})$ of graphene attached on Ni(111) at bridge-top (pink bars) and top-fcc (orange bars) as obtained at PBE level and including any of the studied DFT-D dispersive forces corrections.

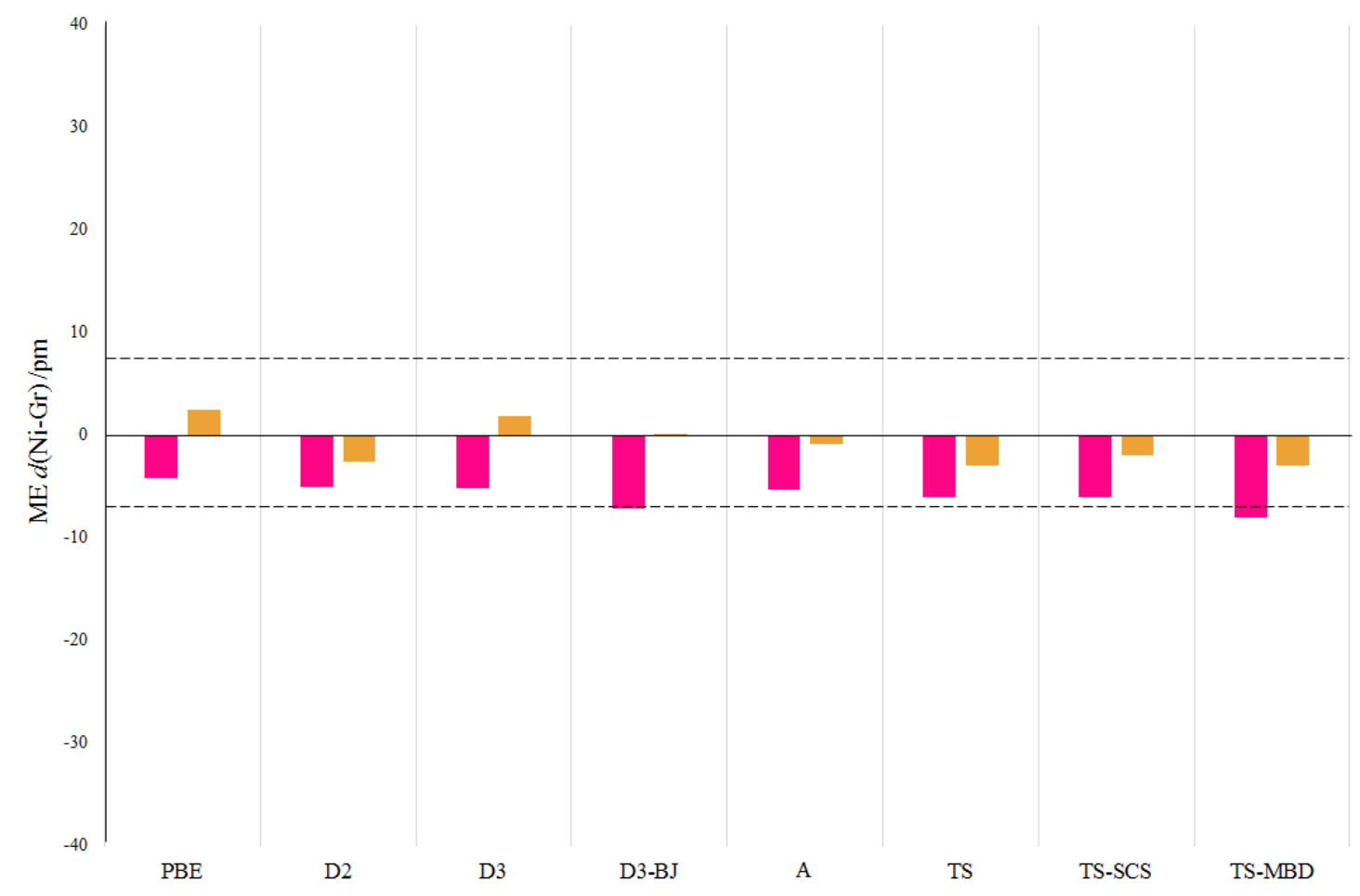


Fig. 4 Bandstructure of graphene adsorbed on Ni(111) in the bridge-top conformation as predicted by PBE. The contributions of graphene $\mathrm{C} s$ and $p$ orbitals to bands are colored violet and pink, respectively. The points in bandstructures whose energy value are analyzed in detail are marked by green circles. Zero energy is here the Fermi energy.

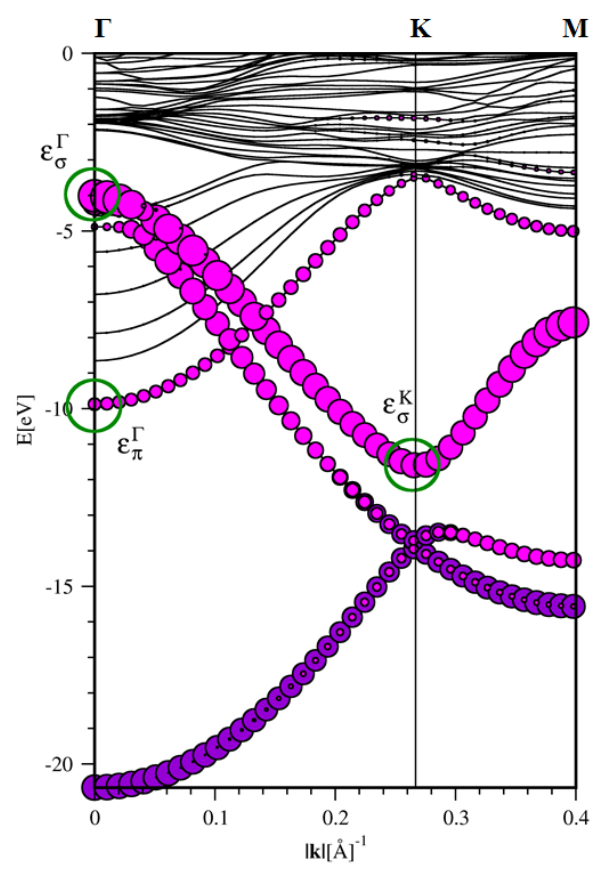


Fig. 5 Mean Error $(M E)$, in $\mathrm{kJ} \mathrm{mol}^{-1}$, for the adsorption energy $E_{a d s}$ of graphene attached to Ni(111) in the top-fcc conformation as obtained with different DFT, DFT-D, vdW-DFT, and RPA methods. Blue bars are present results, whereas green bars are values from previous studies. See Table S1 in Supplementary Material for further details. ${ }^{a}$ Ref. [8], ${ }^{b}$ Ref. [48], ${ }^{c}$ Ref. [55], ${ }^{d}$ Ref. [49], ${ }^{e}$ Ref. [23], ${ }^{f}$ Ref. [56], ${ }^{g}$ Ref. [57], ${ }^{h}$ Ref. [58], ${ }^{i}$ Ref. [50], ${ }^{j}$ Ref. [21], ${ }^{k}$ Ref. [59], ${ }^{l}$ Ref. [46].

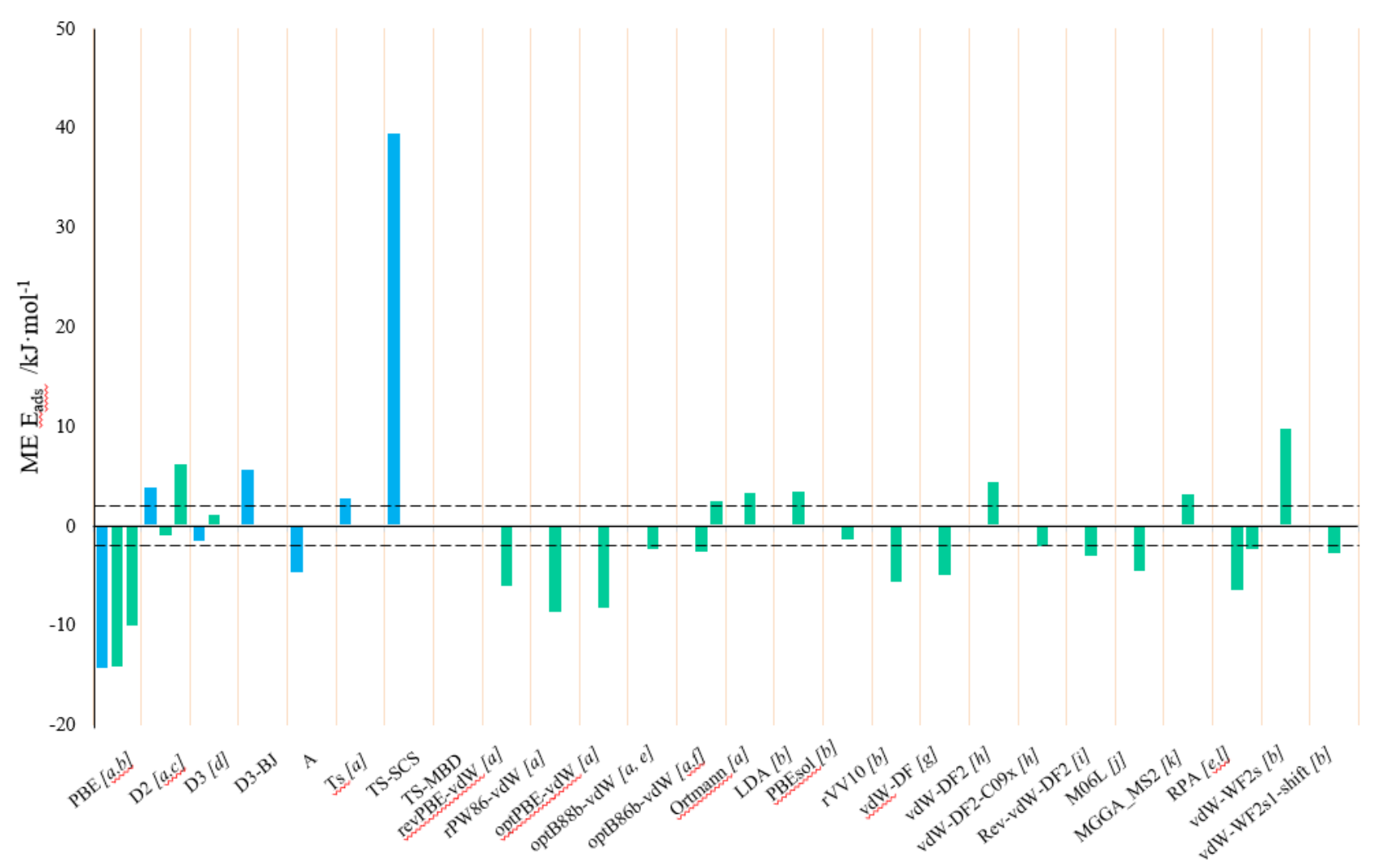


Fig. 6 Mean Error $(M E)$, in pm, for the distance $d(\mathrm{Gr}-\mathrm{Ni})$ of graphene attached to Ni(111) in the topfcc conformation as obtained with different DFT, DFT-D, vdW-DFT, and RPA methods. See Table S1 in Supplementary Material for further details. Orange bars are present results, whereas red bars are values from previous works. References as in Fig. 5.

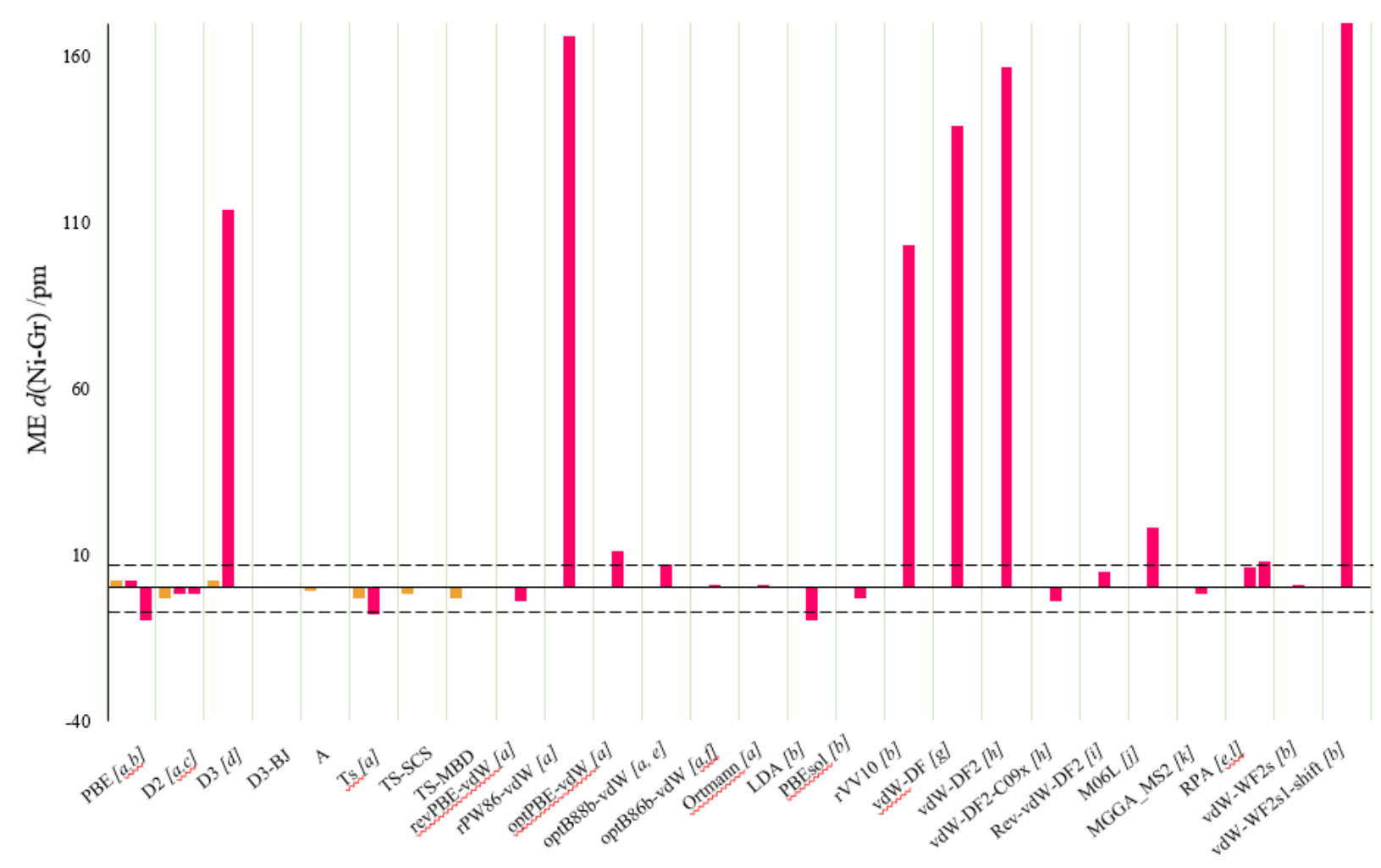




\section{References}

1. Novoselov KS, Geim AK, Morozov SV, Jiang D, Zhang Y, Dubonos SV, Grigorieva IV, Firsov AA (2004) Science 306:666

2. N'Diaye AT, Bleikamp S, Feibelman PJ, Michely T (2006) Phys Rev Lett 97:215501

3. Marchini S, Günther S, Wintterlin J (2007) Phys Rev B 76:075429

4. Vázquez de Parga AL, Calleja F, Borca B, Passeggi Jr. MCG, Hinarejos JJ, Guinea F, Miranda R (2008) Phys Rev Lett 100:056807

5. Koch RJ, Weser M. Zhao W, Viñes F, Gotterbarm K, Kozlov SM, Höfert O, Ostler M, Papp C. Gebhardt J, Steinrück HP, Görling A, Seyller T (2012) Phys Rev B 86:075401

6. Kim KS, Zhao Y, Jang H, Lee SY, Kim JM, Kim KS, Ahn JH, Kim P, Choi JY, Hong BH (2009) Nature 457:706

7. Varyjhalov A, Sánchez-Barriga J, Shikin AM, Biswas C, Vescovo E, Rybkin A, Marchenko D, Reader O (2008) Phys Rev Lett 101:157601

8. Janthon P, Viñes F, Kozlov SM, Limtrakul J, Illas F (2013) J Chem Phys 138:244701

9. Zhao W, Kozlov SM, Höffert O, Gotterbarm K, Lorenz MPA, Viñes F, Papp C, Görling A, Steinrück HP (2011) J Phys Chem Lett 2:759

10. Bianchini F, Patera LL, Peressi M, Africh C, Comelli G (2014) J Phys Chem Lett 5:467

11. Shon NH, Ando T (1998) J Phys Soc. Japan 67:2421

12. Ando T, Zheng Y, Suzuura H (2002) J Phys Soc of Japan 71:1318

13. Uchoa B, Lin CY, Castro Neto AH (2008) Phys Rev B 77:035420

14. Kozlov SM, Viñes F, Görling A (2012) J Phys Chem C 116:7360

15. Gebhardt J, Viñes F, Görling A (2012) Phys Rev B 86:195431

16. Varykhalov A, Marchenko D, Sánchez-Barriga J, Scholz MR, Verberck B, Trauzettel B, Wehling TO, Laubschat C (2008) Phys Rev X 2:107602

17. Mura M, Gulans A, Thonhauser T, Kantorovich L (2010) Phys Chem Chem Phys 12:4759 (2010).

18. Sony P, Puschnig P, Nabok D, Ambrosch-Draxl C (2007) Phys Rev Lett 99:176401

19. Janthon P, Kozlov SM, Viñes F, Limtrakul J, Illas F (2013) J Chem Theory Comput 9:1631

20. Fuentes-Cabrera M, Baskes MI, Melechko AV, Simpson ML (2008) Phys Rev B 77:035405

21. Andersen M, Hornekaer L, Hammer B (2012) Phys Rev B 86:085405

22. Janthon P, Luo S, Kozlov SM, Viñes F, Limtrakul J, Truhlar DG, Illas F (2014) J Chem Theory Comput 10:3832 
23. Mittendorfer F, Garhofer A, Redinger J, Kilmeš J, Harl J, Kresse G (2011) Phys Rev B $84: 201401$

24. Zhang WB, Chen C, Tang PY (2014) J Chem Phys 141:044708

25. Grimme S (2006) J Comput Chem 27:1787

26. Tkatchenko A, Scheffler M (2009) Phys Rev Lett 102:073005

27. Perdew JP, Burke K (1996) Phys Rev Lett 77:3865

28. Kresse G, Furthmüller J (1996) Comput Mater Sci 6:15

29. Note that PAW pseudopotentials released in VASP 5.2 version were used for TS related DFT-D corrections.

30. Monkhorst HJ, Pack JD (1976) Phys Rev B 13:5188

31. Blöchl PE, Jepsen O, Andersen OK (1994) Phys Rev B 49:16223

32. Grimme S, Antony J, Ehrlich S, Krieg S (2010) J Chem Phys 132:154104

33. Grimme S, Ehrlich S, Goerigk L (2011) J Comp Chem 32:1456

34. Andersson MP (2013) J Theo Chem 2013:327839

35. Bučko T, Lebègue S, Hafner J, Ángyán JG (2013) Phys Rev B 87:064110

36. Ambrosetti A, Reilly AM, DiStasio Jr. RA, Tkatchenko A (2014) J Chem Phys 140:18A508

37. Ruiz V, Liu W, Zojer E, Scheffler M, Tkatchenko A (2012) Phys Rev Lett 108:146103

38. For $\mathrm{Ni}, \mathrm{C}^{6}, \alpha$, and $\mathrm{R}_{0}$ values are $59,10.2$, and 2.28 atomic units, respectively.

39. Grimme S (2011) WIREs Comput Mol Sci 1:211

40. Gamo Y, Nagashima A, Wakabayashi M, Terai M, Oshima C (1997) Surf Sci 374:61

41. Shelton JC, Patil HR, Blakely JM (1974) Surf Sci 43:493

42. Zacharia R, Ulbricht H, Hertel T (2004) Phys Rev B 69:155406

43. As for bulk Ni, a $7 \times 7 \times 7$ k-points grid was used in these calculations.

44. Gao W, Tkatchenko A (2015) Phys Rev Lett 114: 096101

45. Bučko T, Lebègue S, Gould T, Ángyán JG (2016) J Phys Condens Matter 28: 045201

46. Olsen T, Thygesen KS (2012) Phys Rev B 87:075111

47. Grüneis A (2013) J Phys Condens Matter 25:043001

48. Silvestrelli PL, Ambrosetti A (2015) Phys Rev B 91:195405

49. Li X, Feng J, Wang E, Meng S, Kilmeš J, Michaelides A (2012) Phys Rev B 85:085425

50. Hamada I (2014) Phys Rev B 89:121103

51. Becke AD (1986) J Chem Phys 85:7184

52. Wellendorff J, Lundgaard KT, Mogelhoj A, Petzold V, Landis DD, Nørskov JK, Bligaard T, Jacobsen KW (2012) Phys Rev B 85:235149 
53. Klimeš J, Bowler DR, Michaelides A (2011) Phys Rev B 83:195131

54 Klimeš J, Bowler DR, Michaelides A (2012) J Phys Condens Matter 22:022201

55. Hasegawa M, Nishidate K, Hosokai T, Yoshimoto N (2013) Phys Rev B 87:085439

56. Zhang WB, Chen C, Tang PY (2014) J Chem Phys 141:044708

57. Vanin M, Mortensen JJ, Kelkkanen AK, Garcia-Lastra JM, Thygesen KS, Jacobsen KW (2010) Phys Rev B 81:081408

58. Hamada I, Otani M (2010) Phys Rev B 82:153412

59. Sun J, Entani S, Fang Y, Haunschild R, Perdew P (2013) Phys Rev Lett 111:106401 